\title{
Erratum to: Differential positive selection of malaria resistance genes in three indigenous populations of Peninsular Malaysia
}

\author{
Xuanyao Liu ${ }^{1}$ Yushimah Yunus ${ }^{2}$ Dongsheng $\mathrm{Lu}^{3} \cdot$ Farhang Aghakhanian $^{4}$. \\ Woei-Yuh Saw ${ }^{5,6} \cdot$ Lian Deng $^{3} \cdot$ Mohammad Ali $^{5} \cdot$ Xu Wang $^{6}$ - Fadzilah Mohd Nor ${ }^{7}$. \\ Thuhairah Abdul Rahman ${ }^{7}$. Shahrul Azlin Shaari ${ }^{7}$ Mohd Zaki Salleh ${ }^{8}$.

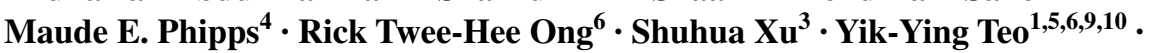 \\ Boon-Peng Hoh ${ }^{2}$
}

Published online: 18 March 2015

(C) Springer-Verlag Berlin Heidelberg 2015

\section{Erratum to: Hum Genet (2015) 134:375-392 DOI 10.1007/s00439-014-1525-2}

Unfortunately, one of the co-author's family name was incorrectly published in the official online publication. The name should appear as Fadzilah Mohd Nor and the correct author group is given below.

Xuanyao Liu - Yushimah Yunus · Dongsheng Lu · Farhang Aghakhanian · Woei-Yuh Saw · Lian Deng · Mohammad Ali · Xu Wang · Fadzilah Mohd Nor · Thuhairah Abdul Rahman - Shahrul Azlin Shaari - Mohd Zaki Salleh • Maude E. Phipps - Rick Twee-Hee Ong · Shuhua Xu . Yik-Ying Teo $\cdot$ Boon-Peng Hoh

The online version of the original article can be found under doi:10.1007/s00439-014-1525-2.

Boon-Peng Hoh

hoh.boonpeng@gmail.com

Yushimah Yunus

statyy@nus.edu.sg

1 NUS Graduate School for Integrative Science and Engineering, National University of Singapore, Singapore 117456, Singapore

2 Institute of Medical Molecular Biotechnology, Faculty of Medicine, Universiti Teknologi MARA, Sungai Buloh Campus, Jalan Hospital, 47000 Sungai Buloh, Selangor, Malaysia

3 Max Planck Independent Research Group on Population Genomics, Chinese Academy of Sciences and Max Planck Society Partner Institute for Computational Biology, Shanghai Institutes for Biological Sciences, Chinese Academy of Sciences Shanghai, Shanghai, China
4 Jeffrey Cheah School of Medicine and Health Sciences, Monash University Sunway Campus, Subang Jaya, Selangor, Malaysia

5 Life Sciences Institute, National University of Singapore, Singapore, Singapore

6 Saw Swee Hock School of Public Health, National University of Singapore, Singapore 117597, Singapore

7 Clinical Pathology Diagnostic Centre Research Laboratory, Faculty of Medicine, Universiti Teknologi MARA, Sungai Buloh Campus, Sungai Buloh, Selangor, Malaysia

8 Integrative Pharmacogenomics Institute, Universiti Teknologi MARA, 42300 Puncak Alam, Selangor, Malaysia

9 Department of Statistics and Applied Probability, National University of Singapore, Singapore, Singapore

10 Genome Institute of Singapore, Agency for Science, Technology and Research, Singapore 138672, Singapore 